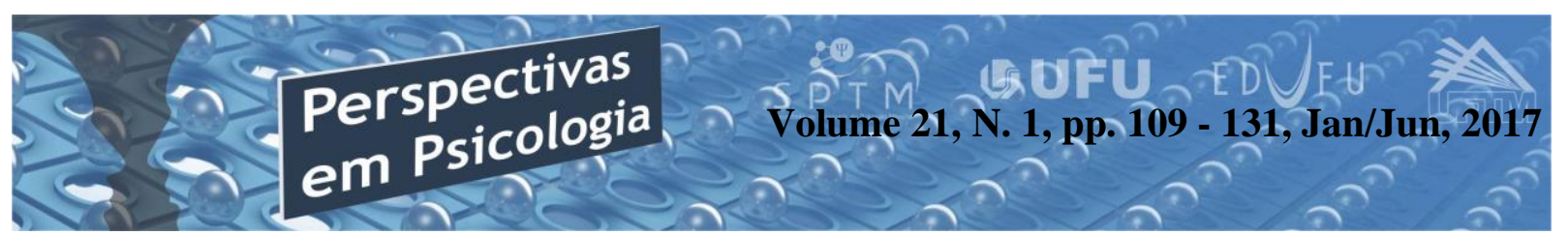

\title{
PSICOLOGIA E CUIDADOS PALIATIVOS: IMPLANTAÇÃO DO SERVIÇO NA UTI DE UM HOSPITAL ESCOLA
}

\author{
Valdir da Silva Júnior \\ Marineia Crosara de Resende \\ (Universidade Federal de Uberlândia - UFU)
}

\begin{abstract}
Resumo
Os Cuidados Paliativos (CP) visa o conforto do paciente, quando sua doença não tem cura. Os CP em UTI é uma prática recente e o objetivo desse trabalho é discorrer sobre a implantação do serviço em CP numa UTI Adulto. Esse serviço foi estruturado com a divisão da equipe em três grupos, cada um com uma tarefa: 1)definir quando incluir o paciente nos $\mathrm{CP}$; 2)estabelecer melhorias na comunicação; 3)ordenar as melhores condutas com o paciente. Nos primeiros seis meses do Serviço implantado foram atendidos 25 pacientes, oito tiveram alta para enfermarias e 17 morreram na UTI, aproximadamente $72 \mathrm{~h}$ após aderir aos CP. A implantação desse Serviço aperfeiçoou o uso de leitos do setor e proporcionou melhorias no trabalho da equipe.
\end{abstract}

Palavras-chave: Psicologia; Cuidados Paliativos; UTI.

\begin{abstract}
Psychology and Palliative Care: Implantation of the Service in ICU of a School Hospital
\end{abstract}

Palliative Care aims at patient's comfort when their diseases don't have cure. In Intensive Care Unit (ICU), palliative care is a recent practice and this work purpose is to discourse about a Palliative Care implantation in adult ICU. The service was structured with the team division in three groups, each one with a specific task: 1) defining when to include a patient in palliative care; 2) establishing communication improvement; 3)order the best course to the patient. In the first six months of implanted service, it where attend 25 patients, witch 8 were discharged for infirmaries and 17 deceased in ICU approximately 72 hours after palliative care adherence. This service implementation perfected sector's beds use and provided teamwork improvement.

Keywords: Psychology; Palliative Care; ICU.

\section{História e Definição dos Cuidados Paliativos}

Os cuidados paliativos apresentamse como uma alternativa para tentar suprir a ineficiência das medidas tecnológicas, 
que não curam e não reestabelecem o quadro de saúde do paciente, igual ao anterior do surgimento da doença, na tentativa de melhorar os cuidados ativos a estes enfermos (Academia Nacional de Cuidados Paliativos [ANCP], 2013). De acordo com os relatos das civilizações ocidentais no passado, a regra era que no momento da morte as pessoas gravemente enfermas permanecessem em suas casas, rodeadas por familiares até o momento do desfecho final (Kübler-Ross, 1998). Assim, os cuidados paliativos despontam como uma tentativa de resgatar tal concepção, em que a morte era considerada como um processo natural, que acontecia de forma confortável, tendo como foco cultural e religioso, o encontro de um significado a essa passagem para a morte, com intuito de dar ênfase na vida que ainda pode ser vivida (Amorim \& Oliveira, 2010; ANCP, 2013).

Por vezes, os Cuidados Paliativos se confundem historicamente com o termo hospice, que definia abrigos (hospedarias) com o propósito de receber e cuidar de peregrinos e viajantes (ANCP, 2013). Em 1840, na França, os hospices recebiam pessoas enfermas e feridas, com risco de morte iminente, durante suas viagens religiosas. Esse movimento de cuidados espalhou-se a outras partes do mundo e, em 1967, foi criado em Londres o St
Cristopher' Hospice, por Cicely Saunders, que revolucionou e incentivou a ideia e criação de hospices independentes, com características de hospitais (ANCP, 2013; Melo \& Figueiredo, 2006).

Em 1970, nos Estados Unidos, Cicely Saunders e Elizabeth Kübler-Ross uniram-se e impulsionaram a continuidade e o crescimento do movimento hospice, que levou o Comitê de Câncer da Organização Mundial de Saúde (OMS) a recomendar, em 1982, que todos os países trabalhassem com esse movimento, visando alívio e cuidados aos pacientes oncológicos (ANCP, 2013). Devido à dificuldade de tradução autêntica em alguns idiomas, o termo hospice foi trocado por Cuidados Paliativos (Conselho Regional de Medicina do Estado de São Paulo [CREMESP], 2008).

$\mathrm{O}$ termo paliativo tem a sua origem no termo latino pallium, que significa “manto, capa." (Twycross, 2003). Em português a noção de Cuidados Paliativos é a tradução literal da expressão inglesa palliative care, usada no Reino Unido desde a década de 80 . O termo paliativo aponta para uma terapêutica que procura minimizar ou eliminar a sintomatologia de uma doença, sem atuar na doença propriamente dita (Abiven, 2001).

Desta forma, nos Cuidados Paliativos a finalidade primária ou 
exclusiva consiste em promover o conforto do paciente, em que os sintomas são encobertos com tratamentos, sem visar a cura. Contudo, esses cuidados não se limitam ao alívio da sintomatologia. Procura-se integrar os aspectos físicos, psicológicos e espirituais, de modo que as pessoas doentes possam se adaptar à esse processo, da própria morte, de forma completa e construtiva quanto seja possível (Twycross, 2003).

Em 1990, a OMS publicou a primeira definição de Cuidados Paliativos como, cuidados totais e ativos dirigidos a pacientes fora de possibilidade de cura. Entretanto, superou-se esse conceito, por ser muito subjetivo o entendimento do momento de identificar que a doença está sem possibilidade de recuperação (CREMESP, 2008).

Assim, em 2002 a OMS recomendou os cuidados paliativos para todos os pacientes com doenças agressivas ou que indiquem risco iminente de morte e não apenas para os casos de câncer (CREMESP, 2008). Além disso, nesse mesmo ano, a OMS (2002) ampliou o conceito deste termo para tipos de cuidados que promovem qualidade de vida de pacientes e de seus familiares diante de doenças sem possibilidades de cura, que ameaçam a continuidade da vida, por meio de prevenção e alívio do sofrimento. Para isso, a OMS (2002) preconiza que a identificação seja precoce e a avaliação e tratamento da dor e outros problemas de natureza física, psicossocial e espiritual sejam efetivos.

A partir dessa definição, os cuidados paliativos pauta-se em alguns princípios fundamentais, como: a) valorizar a obtenção e a manutenção de controle do nível de dor e a administração dos sintomas; b) a consideração do morrer como um processo normal; c) não apressar nem adiar a morte; d) integrar aspectos psicológicos e espirituais aos cuidados do paciente; e) oferecer sistema de apoio para ajudar os pacientes a viver tão ativamente quanto possível, até o momento da sua morte; f) ajudar a família no enfrentamento da doença do paciente e no luto; g) a família também é uma unidade de cuidados; g) abordagem inter e multidisciplinar; h) aprimorar a qualidade de vida; i) são aplicáveis no estágio inicial da doença, concomitantemente com as modificações da doença e terapias que prolongam a vida (Pessini, 2016).

Ao longo das últimas décadas entendeu-se a necessidade de implementação de Cuidados Paliativos para pacientes sem possibilidades terapêuticas de cura (Rezende, Gomes, \& Machado, 2014). O foco da atenção não é a doença a ser curada/controlada, mas a 
pessoa doente, entendida como um ser biográfico, ativo, com direito a informação e a autonomia plena para as decisões a respeito de seu tratamento. A prática adequada dos Cuidados Paliativos preconiza, portanto, atenção individualizada ao paciente e à sua família, busca da excelência no controle de todos os sintomas e prevenção do sofrimento (CREMESP, 2008).

No Brasil, o pioneirismo dos Cuidados Paliativos foi no Rio Grande do Sul (RS), em 1983, onde foi anexado um Serviço de Cuidados Paliativos, junto ao Serviço da Dor do Hospital de Clínicas de Porto Alegre, instituição vinculada à Universidade Federal do Rio Grande do Sul (Figueiredo, 2006). Após esse serviço ser instalado no RS, houve a expansão para o Instituto Nacional do Câncer, localizado no Rio de Janeiro, e depois foi seguido pelos Estados do Paraná, Santa Catarina e São Paulo, na cidade de Jaú, onde foi criada a primeira enfermaria de Cuidados Paliativos no Brasil, em 1992 (Maciel, 2006).

Em 1997, a Associação Brasileira de Cuidados Paliativos, foi fundada no país (Ferrari, Silva, Paganine, Padilha, \& Gandolpho 2008; Maciel, 2006). No ano seguinte, o Instituto Nacional do Câncer, passou a oferecer em um prédio exclusivo, cuidados de enfermaria, pronto- atendimento, serviço ambulatorial e internação domiciliar a pacientes em cuidados paliativos (Silva, \& Silveira, 2015). Em 2002, foi criada pelo Governo Federal a Portaria $n^{\circ}$ 19/ GM de 03 de janeiro de 2002, que institui no Sistema Único de Saúde, o Programa Nacional de Assistência à Dor e Cuidados Paliativos (Brasil, 2002). No ano de 2005, foi fundada a Academia Nacional de Cuidados Paliativos - ANCP, com o intuito de buscar por novas tecnologias, para qualificação de cuidados profissionais e cuidados leigos, incluindo os cuidados paliativos nos programas de atenção à saúde, dos pacientes sem possibilidades de cura, independente da sua enfermidade e idade (Silva \& Silveira, 2015) e nas Unidades de Terapia Intensiva (UTI).

\section{Cuidados Paliativos em UTI}

O Conselho Regional de Medicina do Estado de São Paulo (CREMESP), por meio da Resolução 71/1995, define UTI, em seu artigo $1^{\circ}$, como o local dentro do hospital destinado ao atendimento em sistema de vigilância contínua a pacientes graves ou de risco, potencialmente recuperáveis. $\mathrm{O}$ artigo $2^{\circ}$ desta resolução especifica o "paciente grave" como aquele que apresenta instabilidade de algum de seus sistemas orgânicos, devido a 
PSICOLOGIA E CUIDADOS PALIATIVOS: IMPLANTAÇÃO DO SERVIÇO NA UTI DE UM HOSPITAL

\section{ESCOLA}

alterações agudas ou agudizadas e $\mathrm{o}$ "paciente de risco" como aquele que tem alguma condição potencialmente determinante de instabilidade (CREMESP, 1995, seção 1).

As questões éticas que hoje se apresentam nas UTI são inúmeras e complexas: decisões terapêuticas de investir ou não no tratamento do paciente; definição de estado de reversibilidade ou não; administração de nutrição e hidratação; comunicação de más notícias; participação dos familiares no processo de decisões do paciente; interação profissional da equipe de cuidados que atua na UTI com o paciente e seus familiares; decisões judiciais para admissão de pacientes em UTI, entre tantas outras (Pessini, 2016). A Medicina Intensiva tem sido voraz em incorporar cuidados cada vez mais tecnológicos (Soares, 2007), promovendo uma grande reformulação no conceito de terminalidade da vida, a partir da criação de unidades de terapia intensiva, que disponibilizaram recursos materiais e pessoais com qualificação técnica para o tratamento dos pacientes nesta situação (Martins, 2016).

O desenvolvimento do conhecimento médico, o crescimento das instituições hospitalares e a introdução de inovações tecnológicas trouxeram vários benefícios ao tratamento das mais diversas doenças. Neste aspecto, a evolução da saúde fez com que muitas destas doenças se transformassem em doenças crônicas, proporcionando, consequentemente, o adiamento da morte e longevidade às pessoas com essas enfermidades (Baruzzi \& Ikeoka, 2013).

Neste contexto, chama-se atenção ao fato das questões relacionadas ao fim de vida e à limitação terapêutica, em pacientes com doenças incuráveis e terminais ainda serem negligenciadas. A morte passou a ser evitada a todo custo, com uso de recursos artificiais por períodos indeterminados, em que, pacientes recebem assistência inadequada, quase sempre focada na tentativa de cura, utilizando métodos invasivos e de alta tecnologia (Academia Nacional de Cuidados Paliativos [ANCP], 2013; Baruzz \& Ikeoka, 2013). Esses tipos de abordagens, por vezes, insuficientes, exageradas e desnecessárias, deixam em aberto uma lacuna que se relaciona ao não tratamento do sofrimento e da qualidade de vida do paciente em seu estágio avançado da doença.

Paralelamente observa-se uma crescente preocupação quanto à eficácia e os benefícios dos tratamentos quando utilizados contra a vontade do paciente ou de modo inapropriado, surgindo, assim a pergunta: o ambiente da UTI permite 
prestar cuidados paliativos? (Fonseca, 2012). Os cuidados paliativos são transversais a qualquer prestação de cuidados, revestem-se de uma filosofia de cuidados aplicáveis de forma universal. Logo que a equipe entenda o processo de morrer e aceite a sua inevitabilidade, já estão a desenvolver a capacidade de "estar ao lado" quando a morte chegar (Fonseca, 2012).

Devem iniciar-se, desta forma, os cuidados paliativos junto às técnicas terapêuticas curativas, mesmo que se mantenham os cuidados de índole curativa, fazendo uma transição progressiva entre os dois tipos de cuidados. $\mathrm{O}$ atendimento direciona-se de acordo com o prognóstico da doença, com as possibilidades de tratamento e com os valores e preferências do paciente e de sua família (Baruzzi \& Ikeoka, 2013).

Observa, atualmente, que mesmo nos pacientes em que a cura é utópica, lhes é administrado muitos medicamentos e técnicas invasivas que contribuem, além do aumento nas despesas públicas, para um grande sofrimento físico, psicológico e familiar do paciente (Fonseca, 2012). De um lado, expressões magníficas do progresso técnico-científico da medicina, enquanto de outro, assombra e assusta o fato de se passar por prolongado, sofrido e inútil processo de morte.
No contexto de cuidados intensivos e críticos, sentimentos de esperança a expectativa de uma recuperação “milagrosa”, difícil, mas possível, somados ao medo e angústia profunda de perder a própria vida ou a de alguém querido, estão inacreditavelmente juntos (Pessini, 2016). A necessidade de utilizar medidas paliativas na UTI surgiu a partir de um estudo sobre a elevada prevalência de dor e do controle inapropriado de sintomas, assim como do sofrimento e da angústia, quer seja para o paciente quer seja para a equipe que o assiste (Study to Understand Prognoses and Preferences for Outcomes and Risks of Treatment, 1995).

$\mathrm{O}$ fato de um paciente estar em estado terminal, não significa que, nada mais poderá ser feito em seu benefício, pois existe a possibilidade de lhe serem oferecidas, as condutas que visam aliviar sua dor e desconforto. É exatamente neste estádio terminal da doença, que a relação equipe-paciente, ganha importância para os princípios da bioética (Martins, 2016). A investigação americana Support (1995) identificou, avaliou e elaborou estratégias para melhorar a capacidade de decisão, reduzir a frequência do uso de suporte mecânico, minimizar a dor e evitar o prolongamento do processo do morrer na UTI (Fonseca, 2012). 


\section{ESCOLA}

Do ponto de vista ético e técnico, os profissionais da área da saúde são formados para proporcionarem os melhores cuidados possíveis aos seus pacientes, e assim, é necessário que se promova uma ampla discussão sobre o tema, buscando evitar que as inovações tecnológicas, a pretexto de se prestarem a manter a quantidade de vida, se transformem em instrumentos que prolonguem o sofrimento dos pacientes em estado terminal e que acabem retardando o processo de suas mortes sem qualquer qualidade de vida (Martins, 2016).

Para Bitencourt e outros (2007) continuar utilizando medidas fúteis, em pacientes sem perspectivas de cura, é contra os princípios da ética médica (beneficência, não-maleficência, justiça e autonomia). Esses autores ainda afirmam que continuar um tratamento considerado fútil, não prevê nenhum benefício ao paciente, podendo causar sofrimento e prejuízo ao mesmo, além de utilizar custos e energia que poderiam ser gastos com outro paciente em potencial de recuperação e evitar a morte que é inevitável, sem qualidade de vida, infringindo assim os princípios éticos da medicina (Bitencourt, e outros, 2007). Contudo, ainda existem barreiras ético-culturais e legais, no que se refere a tomada de decisão médica e a adequação da condução do tratamento, ao paciente em estado terminal, submetido a procedimentos na UTI; quer no que se refere a limitação, restringindo os recursos artificiais de suporte de vida; quer ainda, na recusa e suspensão do tratamento (Martins, 2016).

De uma forma geral as ações paliativas na UTI podem ser: a) de planejamento e ação, em que todas as ações de prevenção e terapêuticas devem ser planejadas com a participação da família-paciente e da equipe assistencial; b) privilegiar a adequada comunicação; c) fornecer apoio aos envolvidos no processo, como os familiares e os técnicos; d) permitir a flexibilidade das visitas e, se possível, um acompanhante; e) controle dos sintomas e promoção do conforto ao paciente; f) incorporação da prevenção e tratamento da dor, na rotina dos cuidados intensivos, e o alívio da dor devem ser garantidos, mesmo em situações de duplo efeito de medicação; g) devem ser reconhecidos e tratados os aspectos físicos e psicológicos da dispneia e da dor; h) visar o bem estar do paciente e a não maleficência; através da suspensão de tratamentos fúteis que prolonguem $\mathrm{o}$ morrer, como por exemplo: drogas vasoativas, métodos dialíticos, nutrição parenteral total; i) adequação dos tratamentos não fúteis, como por exemplo: sedoanalgesia individualizada, reavaliação 
do suporte ventilatório (Moritz e outros, 2008; Martins, 2016).

No contexto atual, a implantação de Cuidados Paliativos na UTI está associada a melhor qualidade de vida para os pacientes em estado terminal, uma vez que, acredita-se que esses pacientes não deveriam estar internados e nem morrer nas Unidades de Terapia Intensiva (Martins, 2016). Pensar em Cuidados Paliativos exige necessariamente refletir sobre o trabalho em equipe multiprofissional, seu preparo e os requisitos necessários para essa atuação.

\section{Trabalho Multidisciplinar em Cuidados Paliativos: Enfoque na atuação do Psicólogo}

Os profissionais da área da saúde enfrentam a angústia de muitas vezes não poderem realizar a essência de sua profissão - diagnosticar e curar, o que pode gerar um sentimento de ameaça ao seu saber (Inácio, Vollmann, Langaro, \& Silva, 2015) e certa resistência às mudanças do foco terapêutico, tanto por medo, insegurança, falta de amparo jurídico, quanto pela dificuldade em romper padrões preestabelecidos na formação profissional (Bifulco \& Iochida, 2009). Nesse contexto, o preparo da equipe frente ao paciente sem possibilidades de tratamento de cura é de extrema importância e a comunicação torna-se fundamental para que $o$ atendimento ao paciente e aos seus familiares seja efetivo (Rezende e outros, 2014).

A capacidade de proporcionar um atendimento integral, a escuta ativa, o suporte diante das limitações do adoecimento, as habilidades de comunicações efetivas, conhecimento técnico das situações que irão enfrentar junto ao paciente e a sua família e as estratégias de enfrentamento no que se refere ao fim da vida, são requisitos essenciais para a atuação profissional no contexto de cuidados paliativos. (CREMESP, 2008; Rezende e outros, 2014). Além dos requisitos e habilidades apresentados acima, no que tange à prática, é importante que a equipe realize visitas de acordo com a demanda de cada paciente, a fim de coletar dados que possibilitem discussões sobre sua condição, proporcionando melhores cuidados referentes aos aspectos físicos, sociais e emocionais (CREMESP, 2008).

A informação ao paciente sobre sua doença, além de ser um direito, é essencial, a partir disso, possibilita que ele decida sobre os próximos passos de sua vida e a relação com seus familiares e as pessoas mais próximas (CREMESP, 2008). Por vezes, alguns profissionais, junto aos 
PSICOLOGIA E CUIDADOS PALIATIVOS: IMPLANTAÇÃO DO SERVIÇO NA UTI DE UM HOSPITAL

\section{ESCOLA}

familiares do paciente, acreditam que

As ações paliativas devem valorizar esconder a real situação do mesmo evitará sofrimento, optando assim por não conversar sobre a morte ou a terminalidade. No entanto, quando as dúvidas e os anseios não são compartilhados, cria-se um isolamento emocional, o que faz com que, ao invés de esconder do paciente sua condição, seja necessário que o profissional saiba respeitar o momento de cada paciente, identificando o que ele sabe sobre sua condição e o que ele consegue suportar (Rezende e outros, 2014).

O cuidado paliativo traz desafio ao profissional de saúde, propõe que o cuidado ao paciente seja um equilíbrio entre a competência científica junto à valorização do ser humano. Para que essas necessidades sejam atendidas e o cuidado seja integral é essencial que a equipe de saúde tenha empatia, sendo fundamental ouvir, e que as necessidades dos pacientes sejam priorizadas às habilidades técnicas para diagnosticar e tratar. Pacientes em cuidados paliativos esperam que a relação com os profissionais da saúde seja embasada na compaixão, no respeito e na empatia, de modo a auxiliá-los no processo de morte, valorizando a sua experiência (ANCP, 2012; Oliveira, Santos, \& Mastropietro, 2010) e respeitando sua dignidade. 
Profissionais de saúde especializados ou treinados apresentam melhores resultados no controle de sintomas físicos como a dor e dos sofrimentos psicossociais. A capacitação dessas equipes necessita ser priorizada pelos serviços de saúde (Higginson, \& Evans, 2010). Assistir pessoas em terminalidade mobiliza emoções nos membros da equipe de saúde, pois a morte passa a ser parte do seu cotidiano, gerando conflitos e necessidade de reflexão, sendo necessário um suporte (Cardoso e outros, 2013). É preciso atentar para as necessidades do trabalhador da saúde, como um ser humano que cuida, mas que também precisa ser cuidado, pois esta condição parece influenciar sua disposição para cuidar do próximo, especialmente ao oferecer atenção voltada para os aspectos espirituais (Nascimento, Oliveira, Moreno, \& Silva, 2010).

Em meio a diversas dificuldades nesse processo de fim de vida relacionadas à comunicação, aceitação, enfrentamento, entre outras - faz-se imprescindível a presença do psicólogo na equipe multiprofissional de Cuidados Paliativos. O profissional da psicologia trabalha no sentido de facilitar a compreensão do paciente sobre sua doença e a sua condição de vida, buscando amenizar as dores emocionais e proporcionando conforto para suas angústias, respeitando sempre o seu tempo diante da aceitação da morte que se aproxima (Rezende e outros, 2014).

Ressalta-se a importância da presença do psicólogo desde o momento do diagnóstico, pois sua atuação pode facilitar o ajustamento psicológico do paciente e de sua família e também na comunicação, evitando, por exemplo, a instalação de um silêncio baseado na crença de familiares de que é melhor manter o paciente sem as informações sobre seu estado de saúde (Castro, 2001). O psicólogo trabalha essencialmente com a escuta ativa do paciente, buscando compreender o que o mesmo deseja e, a partir disso, auxiliando a estipular as estratégias de enfrentamento de sua condição como uma pessoa que pensa e sente, mantendo ativas suas opiniões, decisões e o senso de autonomia, o que repercute diretamente no seu bem-estar físico e emocional (Rezende e outros, 2014).

O psicólogo deverá fornecer o suporte necessário para promover a qualidade de vida de pacientes com diagnóstico de doenças sem cura, mantendo o equilíbrio nas suas relações com outros profissionais e encontrando vias de comunicação, que permitam a troca e o conhecimento de diferentes saberes 
(Ferreira, Lopes, \& Melo, 2011). Neste contexto, o vínculo entre o psicólogo e o paciente é crucial para que o trabalho seja eficaz, uma vez que, este auxilia na compreensão da relação entre o paciente e a doença, possibilitando assim a percepção dos sentimentos que surgem junto com as dúvidas e as incertezas durante o tratamento (Rezende e outros, 2014).

A prática do psicólogo com o paciente deve ser orientada no sentido de minimizar o sofrimento inerente a essa fase da vida, buscando a humanização do cuidado; propiciando a comunicação eficaz; a escuta ativa, compreensiva e reflexiva, a elaboração de questões pendentes; facilitando as relações equipe paciente - família, além de uma melhor adesão ao tratamento (Melo, Valero, \& Menezes, 2013). Destaca-se aqui, o papel do psicólogo em dar ênfase e evidenciar o que o paciente já viveu até o momento, contribuindo para que este perceba todas as suas conquistas e os bons momentos vividos (Rezende e outros, 2014) e proporcionando que este viva, independentemente do tempo que ainda lhe resta, uma vida com dignidade, autonomia e entendendo a morte como uma fase natural da vida (Martinho, Pilha, \& Sapeta, 2015).

O acolhimento da família é imprescindível, pois participa desse processo junto ao paciente e vivencia, em alguns momentos, a impotência e os demais sentimentos que a acompanham diante dessa situação (Rezende e outros, 2014). O psicólogo deve proporcionar um espaço de escuta com a família, encontrando meios para evidenciar o cuidado, a qualidade de vida de todos os envolvidos e também desmistificar a possibilidade de não ter mais o que fazer (Maciel, 2006). O trabalho do psicólogo denota apoio emocional aos familiares, a escuta qualificada, o suporte para tomada de decisões, o auxílio na elaboração das vivências relacionadas ao afastamento do paciente, bem como no enfrentamento dos processos de luto (Inácio, Vollmann, Langaro, \& Silva, 2015).

Mesmo o psicólogo fazendo parte da equipe multiprofissional, o que torna necessário que este também encontre um espaço para expressar seus sentimentos e emoções (CREMSP, 2008; Rezende e outros, 2014), cabe a ele a possibilidade de ajuda para a própria equipe que acompanha o paciente nesse processo. A equipe, mesmo capacitada para lidar com a cura, comumente sente-se frustrada, revoltada, fracassada e impotente ao se deparar com pacientes com doenças incuráveis (CREMESP, 2008). A equipe que trabalha com doenças sem perspectivas de cura, pode se beneficiar das intervenções 
psicológicas (Martinho, Pilha, \& Sapeta, 2015), através da mediação da comunicação entre seus membros e também com os familiares, a escuta e o espaço para o reconhecimento das angústias suscitadas pelas demandas de trabalho e a necessidade de produzir aprimoramento em temas como comunicação de más notícias, controle de sintomas e apoio ao luto (Inácio, Vollmann, Langaro, \& Silva, 2015).

Ainda que se observe em vários locais uma falta de preparo nas academias de graduação em relação à temática da morte e do processo de morrer (Bifulco \& Iochida, 2009), a psicologia auxilia nas questões referentes ao enfrentamento de uma notícia de um estado de saúde sem possibilidades de cura, o que ampliou o campo de atuação do psicólogo nas equipes de cuidados paliativos no ambiente de UTI.

\section{Psicologia e Cuidados Paliativos em UTI}

Dentre as diversas funções do psicólogo na UTI estão acompanhar e adaptar as visitas de familiares às rotinas da unidade, preparando os familiares para a entrada, informando as regras que norteiam o bom funcionamento do local (Pregnolatto, \& Agostinho, 2003). O psicólogo deve ainda estimular o contato entre os visitantes e o paciente, observando e avaliando as verbalizações e os comportamentos com a finalidade de verificar a expectativa a respeito do quadro clínico. Para Simonetti (2011), o objetivo da comunicação psicológica com o paciente é, além de passar informações, marcar presença ao lado desse paciente, facilitando a expressão de sentimentos e emoções na tentativa de diminuir a solidão que existe na UTI.

O psicólogo na UTI acompanha pacientes e seus familiares na tentativa de amenizar o sofrimento, a angústia e a solidão nesse momento de intenso desgaste emocional e, também prestar assistência à equipe, permanecendo ao lado, com a finalidade de resgatar a tranquilidade e a sensibilidade para cuidar do próximo, além de propiciar escuta e orientações que se fizerem pertinentes dentro desse contexto (Ferreira \& Mendes, 2013). O trabalho do psicólogo em UTI ajuda também na preparação para o óbito.

Sabe-se que quando os familiares têm a oportunidade de falar sobre a morte e de expressar seus sentimentos, as reações pós-óbito podem se tornar mais amenas e, consequentemente, favorecer uma melhor elaboração do luto. "Preparo para o óbito" vem então como uma tentativa de agregar à Psicologia Hospitalar um novo termo que busca dar suporte emocional aos familiares 
PSICOLOGIA E CUIDADOS PALIATIVOS: IMPLANTAÇÃO DO SERVIÇO NA UTI DE UM HOSPITAL

\section{ESCOLA}

diante desse momento delicado, uma vez A UTI Adulto do HC-UFU

que existe realmente a possibilidade da morte acontecer (Ferreira \& Mendes, 2013), especialmente nos cuidados paliativos.

Os Cuidados Paliativos em UTI é uma prática recente, diante de pacientes terminais, deve-se estabelecer um diferencial no atendimento, focado para o acolhimento das emoções provenientes da possibilidade de morte iminente, da ansiedade pela possibilidade da separação de pessoas queridas, situações e lugares, oferecendo um atendimento humanizado no enfrentamento dessa situação (Haberkorn, 2004). Nesse sentido, o objetivo desse trabalho é discorrer sobre a implantação do serviço em cuidados paliativos na UTI Adulto do HC-UFU.

\section{Método}

\section{A pesquisa}

Os dados foram coletados de um caderno de atas públicas da UTI Adulto do HC-UFU, que discorre sobre o processo e reuniões realizadas para a implantação do Serviço de Cuidados Paliativos no setor. A coleta de dados aconteceu no mês de novembro do ano de 2016. Esses dados serão relatados ao longo do texto.

A equipe de profissionais da UTI Adulto do HC-UFU foi subdividida em

Foram registradas no caderno de atas públicas da UTI Adulto do HC-UFU, um total de treze reuniões, que aconteceram no período de fevereiro de 2015 a março de 2016. As reuniões aconteciam mensalmente e contava com a presença de pelo menos um representante de cada profissão inserida no setor.

Estruturação do Serviço de Cuidados Paliativos na UTI Adulto do HC-UFU 
três grupos para discutirem sobre a temática abordada, que serão descritos no decorrer do texto.

Os profissionais da UTI Adulto do HC-UFU começaram a incluir pacientes no Serviço de Cuidados Paliativos, concomitante à implantação do mesmo. Sendo assim, à medida que o Serviço estava sendo implantada a equipe já incluía o paciente em Cuidados Paliativos quando identificava a impossibilidades de cura da doença.

\section{Resultados e Discussão}

A implantação dos Cuidados Paliativos na UTI Adulto do HCUFU se iniciou, em fevereiro de 2015, devido à mudança nos últimos anos do perfil epidemiológico dos pacientes internados nesse setor. Até então as internações na UTI Adulto do HC-UFU aconteciam primordialmente devido à doenças infectocontagiosas, com grandes possibilidades de recuperação. Entretanto, a predominância das causas de internação dos pacientes passou a ser por doenças crônicas degenerativas, com pouca potencialidade em reverter e curar o quadro clínico. O estudo de Costa Filho, Costa, Gutierrez e Mesquita (2008) aponta que o paliativismo é apropriado para pacientes com câncer, insuficiência cardíaca grave progressiva, falência hepática e/ou renal, doenças neurodegenerativas, lesões medulares graves, doenças pulmonares crônicas e degenerativas, e inúmeras outras condições clínicas encontradas frequentemente nas UTI.

Outra justificava para a implantação do Serviço em Cuidados Paliativos na UTI Adulto do HC-UFU foi o reconhecimento das limitações tecnológicas, que não proporcionava a cura das doenças dos pacientes internados. Consequentemente prolongava-se o período de internação, sem promover a recuperação, acrescendo sofrimento, sem considerar a qualidade de vida. Sendo assim, foram repensadas as formas de cuidado à esses pacientes, na perspectiva de evitar a distanásia - o prolongamento do sofrimento, impedindo a morte a qualquer custo e buscando sempre a ortotanásia evitar as medidas fúteis e proporcionar que a morte aconteça naturalmente, no momento correto e com dignidade (Kovács, 2014).

Para pensar em uma proposta e estruturar o Serviço em Cuidados Paliativos na UTI Adulto do HC-UFU houve a criação de grupos de estudos pelos profissionais do setor sobre a temática, na tentativa de equiparar o conhecimento da equipe.A educação no trabalho é uma das 
PSICOLOGIA E CUIDADOS PALIATIVOS: IMPLANTAÇÃO DO SERVIÇO NA UTI DE UM HOSPITAL

\section{ESCOLA}

estratégias que podem ser utilizadas para possibilitar o desenvolvimento do conhecimento de várias origens (Waterkemper \& Reibnitz, 2010), incluindo-se os cuidados paliativos. $\mathrm{O}$ processo educativo é necessário para garantir a qualidade da assistência nas instituições de saúde, principalmente em situações complexas (Lazari, Schmidt, \& Jung, 2012), como acontece no paliativismo em UTI. Os profissionais do setor UTI do HC-UFU reuniam-se mensalmente, para estudar e discutir sobre essa temática e dividiram-se em três subgrupos.

O primeiro subgrupo tinha o objetivo de definir quando ou não incluir o paciente nos cuidados paliativos. Foi estabelecido um fluxo de decisão de terminalidade, sempre embasados pelo conhecimento científico disponível até o dado momento e contando com as experiências vivenciadas pelos profissionais do setor. Estudos como o de Silva, Souza, Pedreira, Santos e Faustino (2013) e o de Forte (2011) apontam que qualquer pessoa, independente da idade, com diagnóstico de enfermidade sem possibilidade de cura ou com afecção ameaçadora à vida é candidato aos serviços de cuidados paliativos. Sendo assim, a irreversibilidade da doença deverá ser definida de forma consensual pela equipe multiprofissional de saúde que assiste o paciente, baseada em dados objetivos e subjetivos (Silva e outros, 2013; Forte, 2011).

O segundo subgrupo ficou responsável por determinar os métodos de melhoria na comunicação, entre os profissionais da equipe e entre a equipe e familiares/pacientes. A dificuldade de comunicação entre os profissionais de saúde, os pacientes e os familiares é um grande problema nas UTI. A comunicação é considerada como o pilar da Medicina Paliativa, podendo reduzir sintomas rapidamente sem efeitos colaterais, quando realizada de maneira eficaz (Costa Filho e outros, 2008). A boa comunicação em cuidados paliativos é indispensável para que se atenda ao objetivo de cuidar do paciente e do familiar de maneira integral (Sousa \& Carpigiani, 2010).

Ao terceiro subgrupo coube a tarefa de ordenar quais as melhores condutas no manejo com o paciente em cuidados paliativos, listando quais cuidados devem ser mantidos ou não em beneficio do paciente. Silva e outros (2013) apontam que definir quais os cuidados deverão ser mantidos e quais deverão ser suspensos no Serviço em Cuidados Paliativos ainda é um desafio na UTI, em que surgem dúvidas nos integrantes da equipe multiprofissional de saúde como: "Desliga a droga vasoativa 
ou mantém não aumentando a vazão? Mantém a dieta ou não? Aumenta o fornecimento de oxigênio no ventilador caso haja queda de saturação?’(p. 2601).

Todos os subgrupos se pautaram na legislação da bioética vigente para estabelecer os critérios a serem utilizados no Serviço em Cuidados Paliativos na UTI Adulto do HC-UFU, que busca priorizar o consenso entre equipe, família e paciente, quando possível. A inserção e a continuidade dos cuidados paliativos em qualquer setor devem estar embasados em princípios filosóficos que contemplem a bioética, que proporciona um melhor direcionamento para situações que geram dilemas. O respeito aos princípios da bioética garante a excelência dos cuidados paliativos. (Sousa, França, Santos, Costa, \& Souto, 2010).

Nos primeiros seis meses de implantação do Serviço na UTI Adulto do HC-UFU foram atendidos 25 pacientes em Cuidados paliativos, sendo que oito tiveram alta e foram transferidos para as enfermarias, contando com a presença da família por mais tempo na fase final de vida. $\mathrm{E}$ os demais 17 pacientes vieram a óbito na própria UTI, num período aproximado de 72 horas após aderir aos cuidados paliativos. Proporcionar ao paciente em estado terminal, maior contato com a família e o meio externo é de grande importância para o seu conforto e sua qualidade de vida (Silva, Pachemshy, \& Rodrigues, 2009), sendo que esse cuidado se torna mais possível nas enfermarias, por ser ambiente com menos restrições de circulação de pessoas.

$$
\text { A implantação do Serviço em }
$$
Cuidados Paliativos trouxe a otimização no uso dos leitos da UTI Adulto do HC-UFU, no sentido de maior disponibilidade para receber os pacientes com possibilidades de recuperação e ao mesmo tempo não prolongar o sofrimento daqueles sem possibilidades terapêuticas de cura. Proporcionou ainda o fortalecimento e a coesão da equipe, frente ao atendimento do paciente e a temática. A articulação interdisciplinar na equipe é facilitada pela atuação conjunta de profissionais multidisciplinares nas atividades desenvolvidas. A coesão da equipe e as relações profissionais facilitam as trocas entre os diversos saberes, a construção do diálogo e a comunicação mais horizontal entre as diversas profissões. A interdisciplinaridade na área da saúde é um processo em construção em que estão envolvidas as diversas profissões em busca de um objetivo comum, que é a assistência integral ao paciente (Matos, Pires, \& Gelbcke, 2012).

\section{Considerações Finais}




\section{ESCOLA}

Após a implantação do Serviço em Cuidados Paliativos da UTI Adulto do HCUFU, alguns desafios ainda permeiam o setor em relação a este trabalho. Um desses desafios é a continuidade da capacitação dos profissionais do setor, periodicamente, para manter os conceitos e critérios estabelecidos. A qualificação dos profissionais e a produção de conhecimento devem fazer parte de um processo contínuo. A educação continuada busca estimular a consciência crítica, favorecendo espaços para questionamentos, argumentações e possibilidade de continuar aprendendo a desenvolver o cuidado, proporcionando novos saberes que levem à execução adequada do trabalho, permeando a maneira de agir, de compreender e de organizar as condutas no cotidiano (Lazari, Schmidt, \& Jung, 2012).

Outro desafio é a necessidade de implantar os cuidados paliativos nos demais setores do hospital, com a finalidade de evitar que pacientes sem possibilidades de recuperação cheguem até a UTI. As indicações de internação na UTI devem ser analisadas de forma a concluir se haverá maior benefício do que os possíveis sofrimentos ao paciente (Fonseca \& Fonseca, 2010). Além disso, ao ter alta da UTI, o desafio continua, em manter a mesma linha de cuidado com o paciente nas enfermarias, pautado nos critérios estabelecidos em cuidados paliativos consoantes à proposta da UTI Adulto do HC-UFU.

No Brasil, a história dos cuidados paliativos é recente, mas vem conquistando cada vez mais espaço na área da saúde (Cardoso, Muniz, Schwartz, \& Arrieira, 2013; Lima, Almeida, \& Batista, 2014; Silva \& Silveira, 2015). Os cuidados paliativos surgem na tentativa de resgatar a morte como um processo natural da vida, em que pode acontecer de maneira tranquila e sem grandes sofrimentos, visando sempre o bem-estar e a qualidade de vida dos que estão envolvidos nesse processo de viver e morrer (ANCP, 2013), como tem acontecido na UTI Adulto do HC-UFU.

Cuidados Paliativos e UTI, a princípio, parecem ser antagônicos, já que os cuidados paliativos estão diretamente relacionados à preparação para a morte e ao processo de morrer, enquanto a UTI é especializada em reverter casos de pacientes em grave estado de saúde, e manter a vida de pacientes, de maneira artificial até sua recuperação (Waterkemper \& Reibnitz, 2010).

A morte é um tabu a ser desconstruído por todas as categorias profissionais, esse é um assunto que deve 
ser mais discutido e abordado na preparação de futuros profissionais, particularmente na graduação, lócus que prepara os alunos a trabalhar pela vida, sem esquecer que a morte é um episódio que também faz parte dela (Hermes, \& Lamarca, 2013). É preciso clareza aos profissionais da saúde que os Cuidados Paliativos não disputam e nem são incompatíveis com a UTI, ao contrário, sua relevância se dá na maneira com que as ações paliativas poderão auxiliar no plano de cuidado (Fonseca, \& Fonseca, 2010).

Além disso, a mudança no perfil dos pacientes internados em UTI, com impossibilidade de cura das doenças, faz cada vez mais este setor um ambiente propício para adotar os cuidados paliativos, junto aos cuidados curativos. Desta forma, deixando de evitar a morte a qualquer custo e proporcionando uma morte digna, sem prolongamento de sofrimentos desnecessários.

Importante ressaltar a necessidade de novos trabalhos sobre a temática abordada, para aprofundar questões referentes à implantação do Serviço de Cuidados Paliativos na UTI Adulto do HCUFU. Novas pesquisas poderiam ainda, investigar como têm acontecido o trabalho multiprofissional após a implantação desse Serviço, os desafios que ainda permeiam o setor e as conquistas obtidas.

\section{Referências}

Abiven, M. (2001). Para uma Morte mais Humana - Experiência de uma Unidade Hospitalar de Cuidados Paliativos (2a edição). Loures: Lusociência

Academia Nacional de Cuidados Paliativos (ANCP). (2013). Manual de cuidados paliativos. Rio de Janeiro: Diagraphic.

Amorim, W. W.,\& Oliveira, M. G. (2010). Cuidados no final da vida. Saúde Coletiva, 43(7), 198.

Baruzzi, A. C., \& Ikeoka, D. T. (2013). Terminalidade e cuidados paliativos em terapia intensiva. Revista da Associação Médica Brasileira, 59(6), 528-530. https://doi.org/10.1016/j.ramb.2013.06.018

Bifulco, V. A., \& Iochida, L. C. (2009). A formação na graduação dos profissionais de saúde e a educação para o cuidado de pacientes fora de recursos terapêuticos de cura. Revista Brasileira de Educação Médica, 33(1), 92-100. https://doi.org/10.1590/S010055022009000100013 
PSICOLOGIA E CUIDADOS PALIATIVOS: IMPLANTAÇÃO DO SERVIÇO NA UTI DE UM HOSPITAL

\section{ESCOLA}

Bitencourt, A. G. V., el at. (2007). Condutas de limitação terapêutica em pacientes internados em Unidade de Terapia Intensiva. Revista brasileira de terapia intensiva, 19(2), 137-143. https://doi.org/10.1590/S0103-507X2007000200001

Cardoso, D. H., Muniz, R. M., Schwartz, E.,\& Arrieira, I. C. O. (2013). Cuidados paliativos na assistência hospitalar: A vivência de uma equipe multiprofissional. Texto Contexto \& Enfermagem, 22(4), 1134-41. https://doi.org/10.1590/S0104-07072013000400032.

Castro, D. A. (2001). Psicologia e Ética em Cuidados Paliativos. Psicologia ciência e profissão, 21(4),44-51. https://doi.org/10.1590/S1414-98932001000400006.

Conselho Regional de Medicina do Estado de São Paulo (CREMESP). Resolução n 71 de 8 de Novembro de 1995. Diário Oficial do Estado, São Paulo, 1995.

Conselho Regional de Medicina do Estado de São Paulo. (2008). Cuidado Paliativo. São Paulo: CREMESP.

Costa Filho, R. C., Costa, J. L. F., Gutierrez, F. L. B. R., \& Mesquita, A. F. (2008). Como implementar cuidados paliativos de qualidade na unidade de terapia intensiva. Revista Brasileira de Terapia Intensiva, 20(1), 88-92. https://doi.org/10.1590/S0103$507 X 2008000100014$.

Ferreira,P. D., \& Mendes, T. N. (2013). Família em UTI: importância do suporte Psicológico diante da iminência de morte. Revista da SBPH, 16(1), 88-112.

Ferreira, A., Lopes, L., \& Melo, M. (2011). O papel do psicólogo na equipe em cuidados paliativos junto ao paciente com câncer. Revista da Sociedade Brasileira de Psicologia Hospitalar, 14(2), 85-98.

Figueiredo M. T. A. (2006). Reflexões sobre os cuidados paliativos no Brasil. Prática Hospitalar, 8(47), 36-40.

Fonseca, S. F. A. (2012). Relatório prática clínica: Cuidados paliativos em cuidados intensivos Dissertação de Mestrado, Instituto Politécnico de Castelo Branco da Escola Superior de Saúde Dr. Lopes Dias, Castelo Branco, Portugal.

Fonseca, A. C., \& Fonseca, M. J. M. (2010). Cuidados paliativos para idosos na unidade de terapia intensiva: realidade factível. Scientia Medica (Porto Alegre), 20(4), 301-309.

Forte, D. N. (2011). Associações entre as características de médicos intensivistas e a variabilidade no cuidado ao fim de vida em UTI. Tese de Doutorado, Faculdade de Medicina, Universidade de São Paulo, São Paulo, SP. https://doi.org/10.11606/t.5.2011.tde-07122011-124313. 
Haberkorn, A. (2004). A Atuação Psicológica na UTI. Em W. L. Bruscato; C. Benedetti \& S. R. Lopes (Orgs.), A Prática da Psicologia Hospitalar na Santa Casa de São Paulo: Novas páginas em uma Antiga História. (pp. 99-108). São Paulo: Casa do Psicólogo.

Hermes, H. R., \& Lamarca, I. C. A. (2013). Cuidados paliativos: uma abordagem a partir das categorias profissionais de saúde. Ciência \& Saúde Coletiva, 18(9), 2577-2588. https://doi.org/10.1590/S1413-81232013000900012.

Higginson, I. J., \& Evans, C. J. (2010). What is the evidence that palliative care teams improve outcomes for cancer patients and their families. The Cancer Journal, 16(5), 423 35. https://doi.org/10.1097/PPO.0b013e3181f684e5.

Inácio, A. C., Vollman, D. D., Langaro, F., \& Silva, M. C. C. O. (2015). Psicologia e Cuidados Paliativos em UTI neonatal. Revista Interdisciplinar de Estudos em Saúde, 4(2). Retirado de http://www.periodicosuniarp.com.br/ries/article/view/715.

Kluber-Ross E. (1998). A Roda da vida (5a. ed.). Rio de Janeiro: Sextante.

Kovács, M. J. (2014). A caminho da morte com dignidade no século XXI. Revista Bioética, 22(1), 94-104. https://doi.org/10.1590/S1983-80422014000100011.

Lazzari, D. D., Schmidt, N., \& Jung, W. (2012). Educação continuada em unidade de terapia intensiva na percepção de enfermeiras. Revista de Enfermagem da UFSM, 2(1):88-96.

Maciel M. G. S. (2006). A terminalidade da vida e os cuidados paliativos no Brasil: considerações e perspectivas. Prática Hospitalar, 7(47), 46-49.

Maluf, A. C. M., Silva, L., Paganine, M. C., Padilha, K. G., \& Gandolpho, M. A.(2008). Uma leitura bioética sobre cuidados paliativos: caracterização da produção científica sobre o tema. Bioethikos, 2(1), 99-104.

Martins, B. C. P. C. C. (2016). Cuidados Paliativos para pacientes em estado terminal em Unidades de Terapia Intensiva, Revisão Sistemática e Metanálise. Dissertação de Mestrado, Faculdade de Medicina, Universidade Estadual Paulista "Júlio de Mesquita Filho", Botucatu, SP.

Martinho, A. R., Pilha, L., \& Sapeta, P. (2015). Competências do psicólogo em cuidados paliativos. Retirado dehttp://repositorio.ipcb.pt/bitstream/1/RSL\%20repositorio.pdf

Matos, E., Pires D. E. P., \& Gelbcke, F. L. (2012). Implicações da interdisciplinaridade na organização do trabalho da enfermagem: estudo em equipe de cuidados paliativos. $\begin{array}{lllll}\text { Revista } & \text { Eletrônica } & \text { 230-239. }\end{array}$ https://doi.org/10.5216/ree.v14i2.13237. 
PSICOLOGIA E CUIDADOS PALIATIVOS: IMPLANTAÇÃO DO SERVIÇO NA UTI DE UM HOSPITAL

\section{ESCOLA}

Melo, A. C., Valero, F. F., \& M, Menezes. (2013). A intervenção psicológica em cuidados paliativos. Psicologia, Saúde \& Doenças, 14(3), 452-469.

Moritz, R. D. (2008). Terminalidade e Cuidados na unidade de terapia intensiva. Revista Brasileira de Terapia Intensiva, 20(4), 422-428. https://doi.org/10.1590/S0103$507 X 2008000400016$.

Nascimento L. C., Oliveira F. C. S., Moreno M. F., \& Silva F. M. (2010). Cuidado espiritual: componente essencial da prática da enfermeira pediátrica na oncologia. Acta Paulista de Enfermagem, 23(3), 437-40. https://doi.org/10.1590/S0103-21002010000300021.

Nunes, L. V. (2009). Papel do psicólogo na equipe de cuidados paliativos. IN: ANCP. Manual de cuidados paliativos. Rio de Janeiro: Diagraphic.

Oliveira, E. A., Santos, M. A., \& Mastropietro, A. P.(2010). Apoio psicológico na terminalidade: ensinamentos para a vida. Psicologia em Estudo, 15(2), 235-44. https://doi.org/10.1590/S1413-73722010000200002.

Oliveira, A. C., \& Silva, M. J. P. (2010). Autonomia em cuidados paliativos: conceitos e percepções de uma equipe de saúde. Acta paulista de enfermagem, 23(2),212-17. https://doi.org/10.1590/S0103-21002010000200010.

Paiva, F. C. L., Almeida Júnior, J. J., \& Damásio, A. C. (2014). Ética em cuidados paliativos: concepções sobre o fim da vida. Revista Bioética, 22(3), 550-560. https://doi.org/10.1590/1983-80422014223038.

Pessini, L. (2016). Vida e morte na UTI: a ética no fio da navalha. Revista Bioética, 24(1), 54-63. https://doi.org/10.1590/1983-80422016241106.

Portaria ${ }^{\circ}$ 19/ GM de 03 de janeiro de 2002. Institui no Sistema Único de Saúde o Programa Nacional de Assistência à Dor e Cuidados Paliativos. Brasília, DF.

Pregnolatto, A. P. F., \& Agostinho, V. B. M. (2003). O Psicólogo na Unidade de Terapia Intensiva - Adulto. Em M. N. Baptista \& R. R. Dias (Orgs.), Psicologia Hospitalar: Teoria, Aplicações e Casos Clínicos. Rio de Janeiro: Guanabara.

Rezende, L. C. S., Gomes, C. S., \& Machado, M. E. C. (2014). A finitude da vida e o papel do psicólogo: perspectivas em cuidados paliativos. Revista Psicologia e Saúde, 6(1), 2836.

Ribeiro, E. E. (2008). Tanatologia: vida e finitude. Rio de Janeiro: Unati.

Simonetti, A. (2011). Manual de Psicologia Hospitalar. O Mapa da Doença. São Paulo: Casa do Psicólogo. 
Silva, C. F., Souza, D. M., Pedreira, L. C., Santos, M. R., \& Faustino, T. N. (2013). Concepções da equipe multiprofissional sobre a implementação dos cuidados paliativos na unidade de terapia intensiva. Ciência \& Saúde Coletiva, 18(9), 2597-2604. https://doi.org/10.1590/S1413-81232013000900014.

Silva, F. S., Pachemshy, L. R., \& Rodrigues, I. G. (2009). Percepção de enfermeiros intensivistas sobre distanásia em unidade de terapia intensiva. Revista Brasileira de Terapia Intensiva, 21(2), 1982-4335. https://doi.org/10.1590/S0103$507 \times 2009000200006$.

Silva, D. I. S., \& Silveira D. T. (2015). Cuidados paliativos: desafio para a gestão e políticas em saúde. Revista Eletrônica Gestão \&Saúde,6(1),501-513.

Soares, M. (2007). Cuidando da Família de Pacientes em Situação de Terminalidade Internados na Unidade de Terapia Intensiva. Revista Brasileira de Terapia Intensiva, 19(4), 481-484. https://doi.org/10.1590/S0103-507X2007000400013.

Sousa, A. T. O., França, J. R. F. S., Santos, M. F. O., Costa, S. F. G., \& Souto, C. M. R. M. (2010). Cuidados paliativos com pacientes terminais: um enfoque na Bioética Cuidados paliativos. Revista Cubana de Enfermerìa, 26(3), 117-129.

Sousa, K. C., \& Carpigiani, B. (2010). Ditos, não ditos e entreditos: a comunicação em cuidados paliativos. Psicologia: teoria e prática, 12(1), 1516-3687.

The Support principal investigators: a controlled trial to improve care for seriously ill hospitalized patients (1995). JAMA, 274(20), 1591- 1598.

Twycross, R. (2003). Introducing palliative care (4a. ed). Oxford: Radcliffe Medical Press.

Waterkemper, R., \& Reibnitz K. S. (2010). Cuidados paliativos: a avaliação da dor na percepção de enfermeiras. Revista Gaúcha de Enfermagem, 31(1), 84-91. https://doi.org/10.1590/S1983-14472010000100012.

World Health Organization (2002). Definição de cuidados paliativos. Retirado de http://www.who.int/cancer/palliative/definition/en/

World Health Organization. (1990). Cancer Pain Relief and Palliative Care: report of a WHO Expert Committee. Geneva: World Health Organization. Retirado de http://apps.who.int/iris/bitstream/10665/39524/1/WHO_TRS_804.pdf.

\section{Os autores:}


PSICOLOGIA E CUIDADOS PALIATIVOS: IMPLANTAÇÃO DO SERVIÇO NA UTI DE UM HOSPITAL

ESCOLA

Valdir da Silva Júnior possui graduação em Psicologia pela Universidade Federal do Triângulo Mineiro (2014). Atualmente é psicólogo residente da Universidade Federal de Uberlândia. E.mail: valdirjuniors@hotmail.com

Marineia Crosara de Resende. Professora Adjunto do Instituto de Psicologia; Tutora no Programa em Residência Multiprofissional do Hospital de Clínicas da Universidade Federal de Uberlândia.

\section{Endereço para correspondência:}

Prof. Dra. Marineia Crosara de Resende

Instituto de Psicologia da Universidade Federal de Uberlândia

Av. Pará, 1720, bloco 2C, Campus Umuarama

CEP: 38405-382 - Uberlândia - MG.

E-mail: marineia@ipsi.ufu.br

Recebido em: 06/06/2017.

Aprovado em: 23/06/2017 